\title{
A multiscale finite element simulation of piezoelectric materials using realistic crystal morphology
}

\author{
Y. Uetsuji ${ }^{1}$, H. Kuramae ${ }^{2}$, K. Tsuchiya ${ }^{3}$ \& M. Kamlah ${ }^{4}$ \\ ${ }^{I}$ Department of Mechanical Engineering, \\ Osaka Institute of Technology, Japan \\ ${ }^{2}$ Department of Technology Management, \\ Osaka Institute of Technology, Japan \\ ${ }^{3}$ Department of Precision Engineering, Tokai University, Japan \\ ${ }^{4}$ Institute for Materials Research II, \\ Karlsruhe Institute of Technology, Germany
}

\begin{abstract}
This paper presents the full components of macroscopic homogenized material properties and the microscopic localized response obtained through a multiscale finite element simulation using realistic crystal morphology. Crystal morphology analysis was performed to reveal microstructure and texture of a polycrystalline piezoelectric material. The insulative specimen of piezoelectric material was coated with a conductive layer of amorphous osmium to remove an electric charge, and crystal orientations were measured by means of electron backscatter diffraction. Then the obtained crystal orientations were applied to a multiscale finite element simulation based on homogenization theory.

Keywords: piezoelectric material, EBSD, crystal morphology, multiscale finite element simulation, homogenization theory.
\end{abstract}

\section{Introduction}

Piezoelectric materials have been used in actuators or sensors as a component of various electronic and mechanical devices. Generally these materials consist of many crystal grains and domains at a microscopic scale. Since each domain shows strongly anisotropic mechanical and electrical behaviours according to 
asymmetrical crystal structure such as tetragonal and rhombohedron ones, the macroscopic properties of polycrystalline piezoelectric materials have a large dependence of the microscopic crystal morphology. Therefore, it is important to understand the microstructural features. Scanning electron microscope (SEM) and electron backscatter diffraction (EBSD) technique [1, 2] is well-established and useful to characterize the crystal morphology in polycrystalline materials. Electron beam is irradiated to a specimen in SEM, and then crystal orientations of sub-micron order grains and domains can be analyzed by EBSD. SEM - EBSD technique is hitherto applied to various materials, which range from metals [3, 4] to functional materials [5]. Especially if we focus on piezoelectric materials, a lot of published literatures have reported their microstructures and textures for lead titanate $\mathrm{PbTiO}_{3}$ [6], barium titanate $\mathrm{BaTiO}_{3}$ [7] and $\mathrm{PZT} \mathrm{Pb}(\mathrm{Zr}, \mathrm{Ti}) \mathrm{O}_{3}$ [8]. All of them indicate that the microstructures have a significant role in the overall material properties. However, there are few researches to analyze the quantitative relationship between the microstructure and the macrostructure.

On the other hand, we employ a multiscale finite element simulation based on homogenization theory [9-11] and attempt to estimate the relationship of SEM $\cdot$ EBSD-measured realistic microstructure with material properties and behaviours of piezoelectric materials. In case of our previous study on $\mathrm{BaTiO}_{3}$ [12], crystal orientation maps with high resolution couldn't be obtained because of the electrification of specimen. Additionally, in our previous investigation on PZT [13], the computations were carried out by using uncertain material properties to microstructural grains because single crystals are nonexistent and their material properties were unknown. In this paper, we retry to analyze the microstructure of $\mathrm{BaTiO}_{3}$ by SEM $\cdot$ EBSD measurement. With the aim of a high resolution crystal orientation map, a conductive layer of amorphous osmium is utilized to remove an electric charge on the specimens. Then we employ a multiscale finite element simulation and investigate on the macroscopic homogenized material properties developed by SEM -EBSD-measured realistic microstructure and the microscopic localized behaviours in response to macroscopic external load.

\section{Crystal morphology analysis}

A $\mathrm{BaTiO}_{3}$ polycrystalline piezoelectric ceramics (Murata Manufacturing Co. Ltd.) was used for the SEM-EBSD specimen. As shown in figure 1, the specimen was a circular disk of $15 \mathrm{~mm}$ diameter and $1 \mathrm{~mm}$ thickness, and it was electrically poled along the thickness direction. The crystal structure was examined by means of X-ray diffraction (XRD). Figure 2 shows XRD spectrum of $\mathrm{BaTiO}_{3}$ piezoelectric material. It has a perovskite tetragonal structure and the lattice constants of $a$ and $c$ axes are $0.3995 \mathrm{~nm}$ and $0.4034 \mathrm{~nm}$, respectively. 


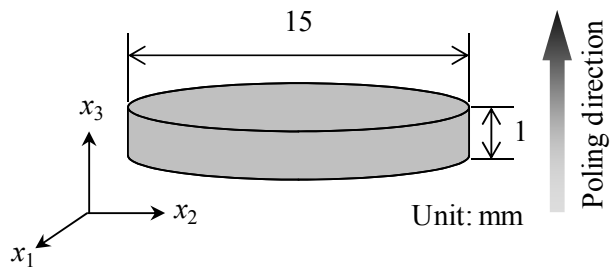

Figure 1: Geometry of SEM $\cdot$ EBSD specimen.

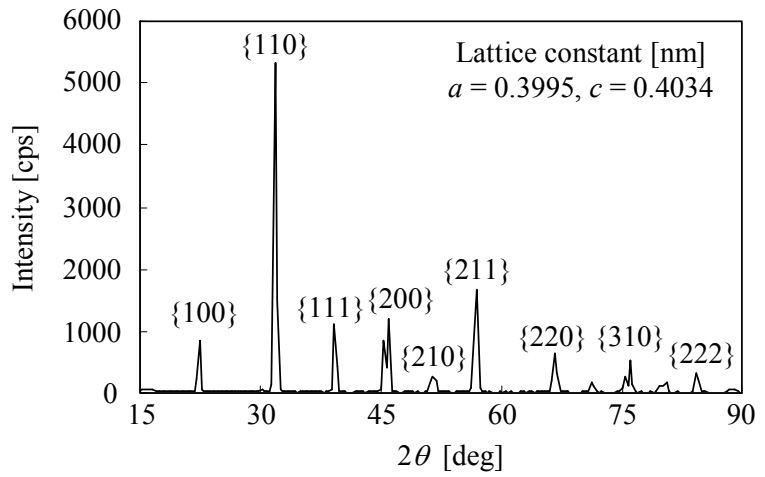

Figure 2: $\quad$ XRD spectrum of $\mathrm{BaTiO}_{3}$ piezoelectric ceramics.

The observed surface was mechanically polished for 20 minutes using $3 \mu \mathrm{m}$ diamond particles (Marumoto Struers Co., DP-Spray) with a polishing sheet (Marumoto Struers Co., DP-Mol). And then, it was chemically polished at $\mathrm{pH}$ 3.5 for 20 minutes using colloidal particles (Marumoto Struers Co., OP-A) with a polishing sheet (DP-Chem). The surface of the insulative specimen was coated with the electrical conductive and amorphous osmium layer to defend the electrification due to electron beam. We employed an osmium coater (Meiwafosis Co., LTD. Neoc-ST), and set the discharging time in osmium gas to 10 seconds. The estimated thickness of osmium layer is $0.5 \mathrm{~nm} / \mathrm{sec}$. In addition, a silver paste (Fujikura Kasei Co. Ltd., type D-500) was applied to the surface except for the SEM-EBSD measurement region to leak the accumulated charge.

Figure 3 shows crystal morphology images obtained by means of EBSD (Oxford Instruments plc, Link ISIS C.7272) implemented in SEM (JEOL Datum Ltd., JSM-5410). The scanning interval was set to $0.64 \mu \mathrm{m}$ that is smaller than one over ten of the average grain size. As shown in figure 4, crystal orientations in four neighboring regions of $81.3 \times 63.5 \mu^{2}$, which was the obtainable maximum region at once, were measured successively. Their four-divided crystal orientation maps were spliced to keep their exact spatial arrangement. As a 
result, a crystal orientation map of $127.6 \times 90.8 \mu \mathrm{m}^{2}$ was obtained as shown in figure 4 . The mean diameter of grain was estimated to approximately $6.71 \mu \mathrm{m}$.

Figures 3(a) and 4 are the collar map of crystal orientations, and figure 3(b) is the grayscale map of pattern q1uality (PQ) value defined by eqn. (1).

$$
\mathrm{PQ}=\sum_{i=1}^{3} h_{i} / 3 \sigma_{h}
$$

where $h_{i}$ means the peak height of the Hough transformed $i$-th Kikuchi's band. $\sigma_{h}$ is the standard deviation of Hough transform. The PQ value represents quantitatively the fitting condition of Kikuchi patterns with the target crystal

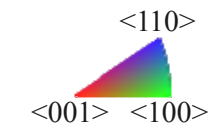

(i)

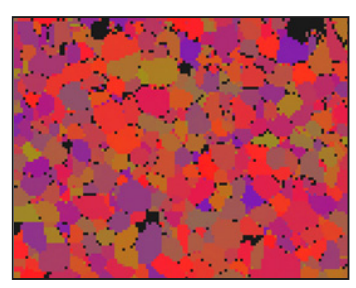

(ii)

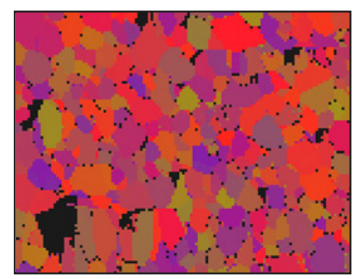

(iii)

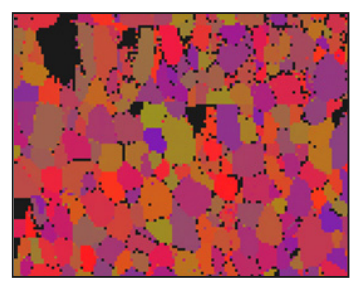

(iv)

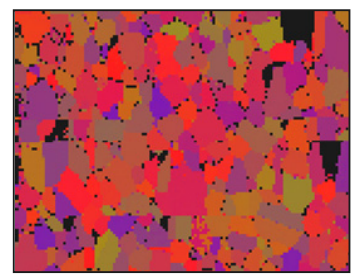

(a) Orientation map
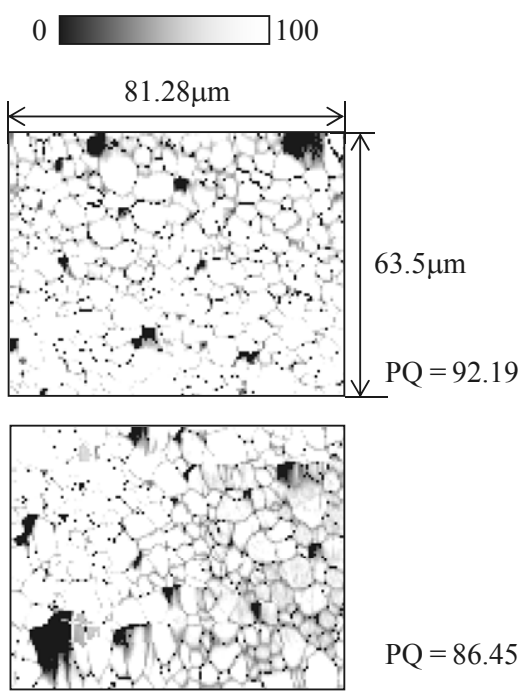

$\mathrm{PQ}=86.45$

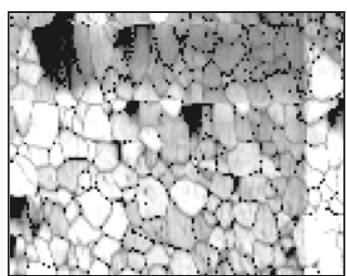

$\mathrm{PQ}=69.34$

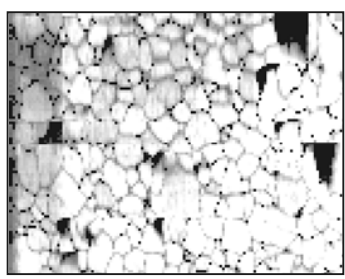

$\mathrm{PQ}=80.88$

(b) PQ map

Figure 3: Crystal orientation and PQ maps of $\mathrm{BaTiO}_{3}$ piezoelectric ceramics. 
structure. On grain boundaries, where Kikuchi pattern may be composed of two or more orientations from adjacent grains, the PQ parameter yielded low value. Additionally, the reduction of PQ value appeared at the overlapping regions of scanning electron beam as demonstrated in (iii) of figure 3.

The crystal orienting degree in eqn. (2) was employed for an indicator to catch the orientation of domains toward an optional direction.

$$
S_{y_{1^{\prime}}}^{c}=\frac{\sum_{i=1}^{n}\left|\boldsymbol{e}_{c}^{i} \cdot \boldsymbol{e}_{y_{1^{\prime}}}\right|}{n}
$$

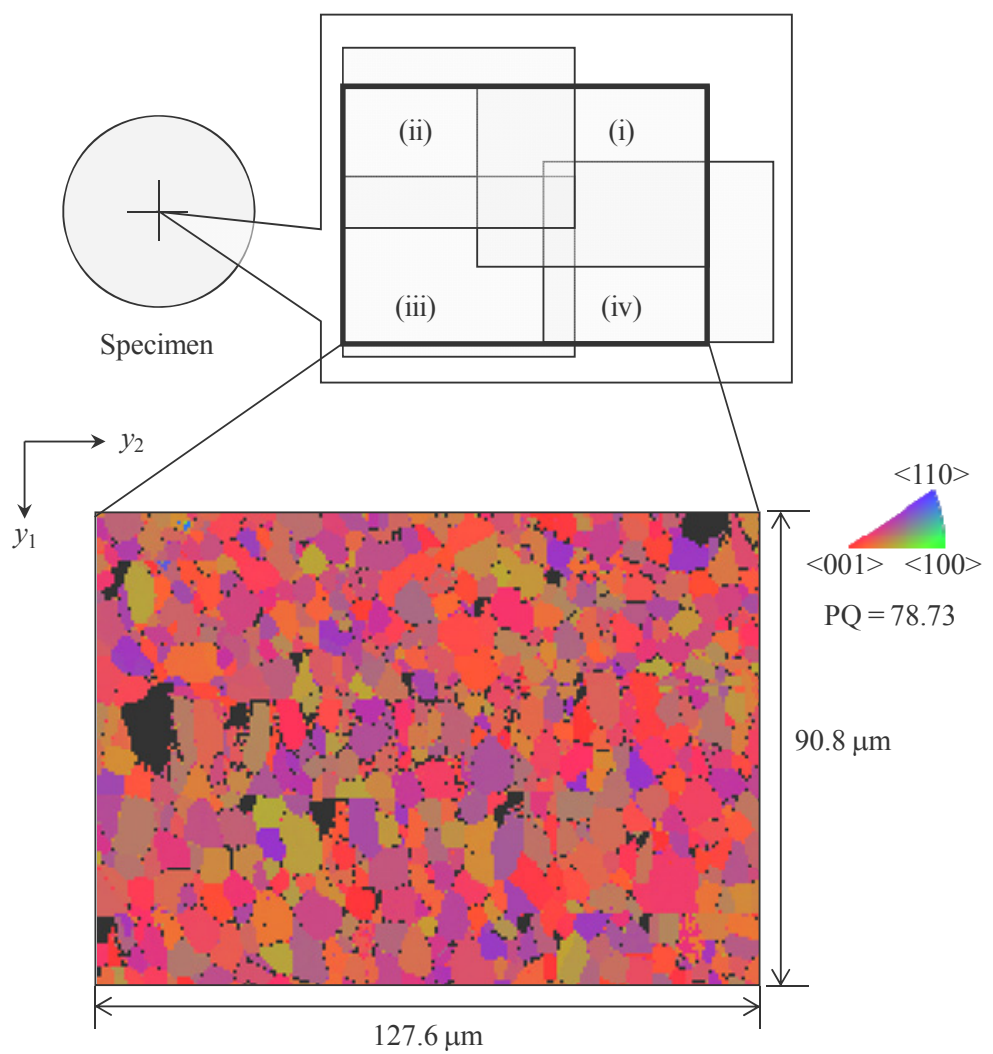

Figure 4: $\quad$ Spliced crystal orientation map of $\mathrm{BaTiO}_{3}$ piezoelectric ceramics.

where $\boldsymbol{e}_{c}^{i}$ means the basis vector of $c$ axis of the crystallographic coordinate system in microstructural coordinate system at the $i$-th measuring point. $\boldsymbol{e}_{y_{1}}$ is the basis vector of an optional direction $y_{1}$ ' existing in the $y_{1}-y_{2}$ plane. And $n$ is the total number of measuring points. The crystal orienting degree becomes zero when all points orient to the normal direction of the $y_{1}-y_{2}$ plane and it becomes 


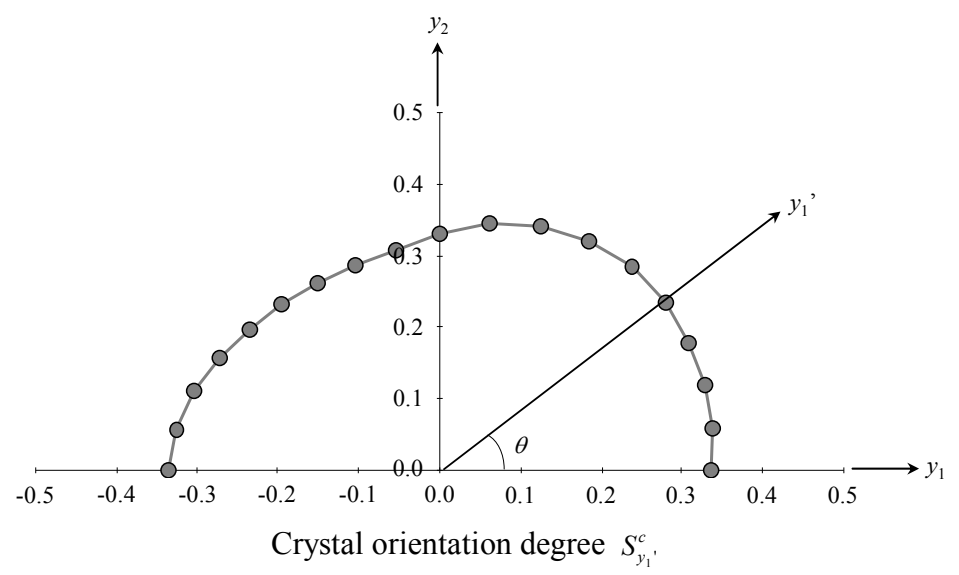

Figure 5: $\quad$ Inplane crystal orienting degree of microstructure.

one when all points orient to $y_{1}$ ' direction. Figure 5 shows the crystal orienting degree for the crystal orientation map in figure 4 . These values were calculated through changing the angle $\theta$ between $y_{1}$ and $y_{1}$ ' from $0^{\circ}$ to $180^{\circ}$. The crystal orienting degree indicated a multi-directionally uniform orientation in the $y_{1}-y_{2}$ plane.

\section{Multiscale finite element simulation}

A three-dimensional multiscale finite element simulation based on homogenization theory was employed to investigate the relationship between $\mathrm{SEM} \cdot \mathrm{EBSD}$-measured realistic microstructure and macrostructure. Figure 6 illustrates the scheme of multiscale modelling for polycrystalline piezoelectric materials. The details of formulations and computational approach were presented in the published literatures [10, 11]. A macrostructure is enough large compared with microstructures and it can be assumed that microstructures have the periodicity at microscopic scale. In that case, the multiscale simulation based on homogenization theory enables us estimate the macrostructural homogenized material properties from heterogeneous microstructures. And it also enable us evaluate the microscopic mechanical and electrical behaviours in response to the external load applied to macrostructure.

Figure 7 shows the finite element modelling of SEM-EBSD-measured realistic microstructure. The crystal orientations in $90.8 \times 90.8 \mu^{2}$, which consisted of approximately 233 grains, were sampled from the central region of $127.6 \times 90.8 \mu \mathrm{m}^{2}$ in figure 4 . A plate-shaped regular cubic mesh, whose number of elements corresponds exactly to one of measuring points, was employed for a microstructural finite element model. The crystal orientation of each measuring point was mapping independently into one element. Our previous investigation 


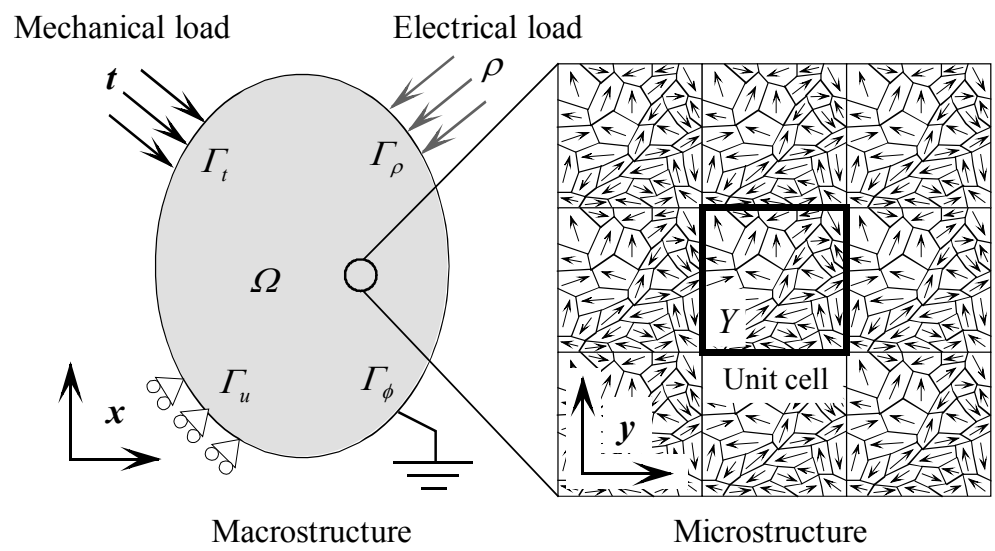

Figure 6: Multiscale modelling of polycrystalline piezoelectric materials.
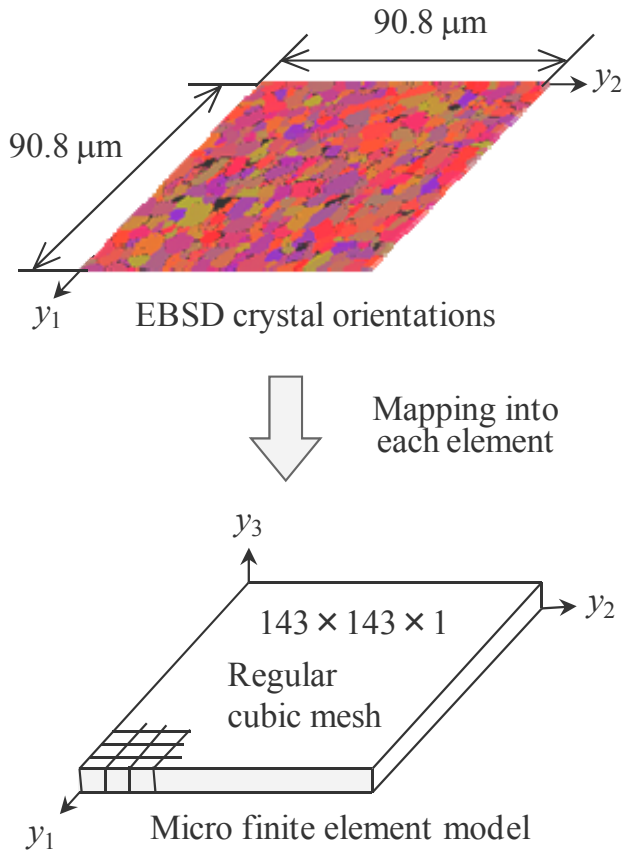

Figure 7: $\quad$ FE modelling of SEM $\cdot$ EBSD-measured realistic microstructure.

for PZT [13] indicated that the dependence on the sampling area of crystal orientations falls off and the relative error of macroscopic homogenized material properties becomes $1 \%$ or less if the number of grains is beyond approximately 200. Consequently, the crystal orientation map of $90.8 \times 90.8 \mu \mathrm{m}^{2}$ can be regards as a representative volume element of microstructure since it includes 233 grains. 
Figure 8 shows the full components of macroscopic homogenized material properties obtained through the multiscale simulation using the SEM EBSDmeasured realistic microstructure in figure 7. The material properties of $\mathrm{BaTiO}_{3}$ single crystal [14] were utilized for each element on the assumption that each grain is poled fully and it consists of a single domain. Figure 8 (a), (b) and (c) indicate the elastic compliance constants, relative dielectric constants and piezoelectric strain constants, respectively. Figure 8 (c) includes the experimental values described in a brochure provided by Murata Manufacturing Co. Ltd. The computational values of piezoelectric strain constants have some correspondence to experimental values although ${ }^{\text {macro }} d_{33}$ has relatively-large error more than $20 \%$. If we focus on the transverse isotropy, good agreements can be confirmed between the components relating to inplane first and second axes.

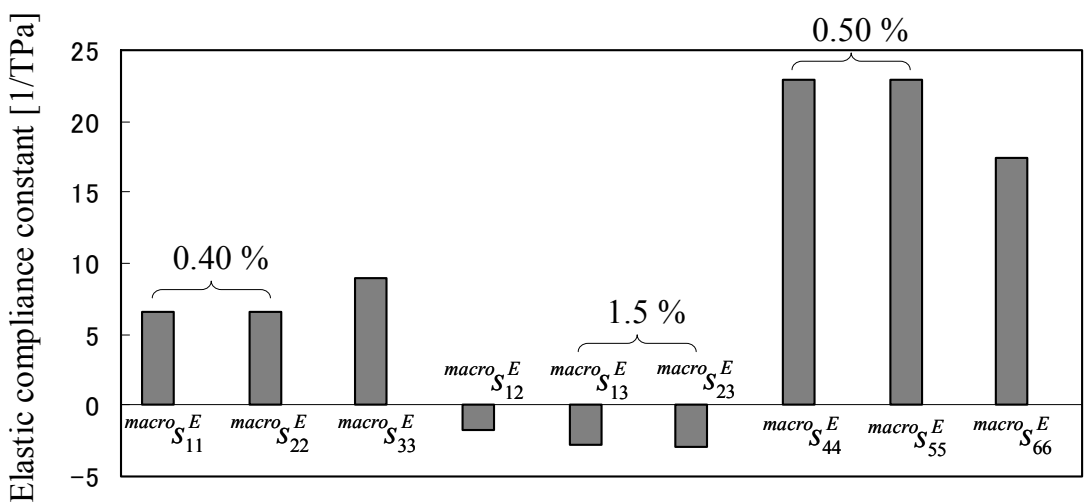

(a) Elastic compliance constants

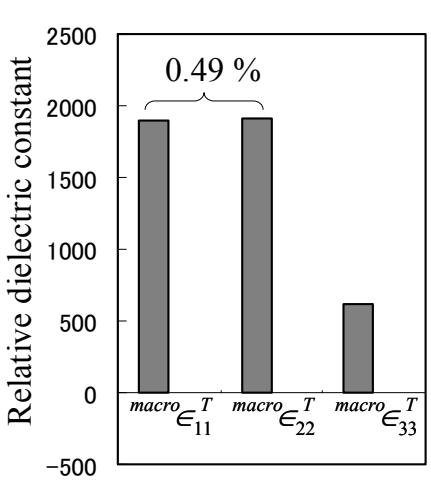

(b) Relative dielectric constants

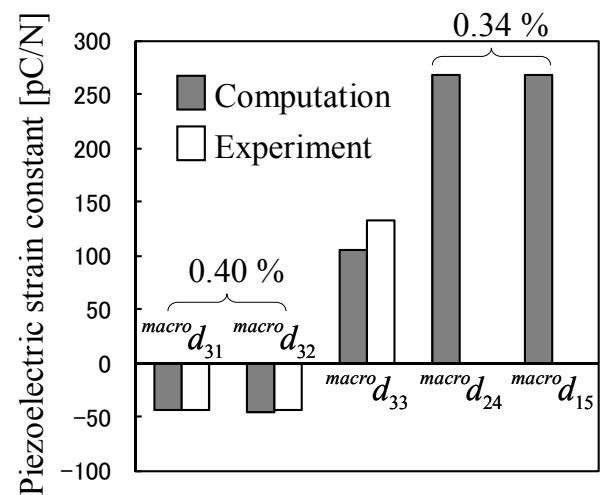

(c) Piezoelectric strain constants

Figure 8: The full components of macroscopic homogenized material properties obtained through the multiscale finite element simulation using SEM·EBSD-measured realistic microstructure. 
And now, we discuss the microscopic localized behaviours of SEM-EBSDmeasured realistic microstructure in response to a macroscopic external load. Figure 9 (a) demonstrates the macrostructure and its boundary conditions, and (b) shows the microstructure. While the above-mentioned $143 \times 143 \times 1$-divided mesh was used for microstructure, a one-element mesh was employed for macrostructure because of a linear displacement and electrical potential field. The macroscopic homogenized material properties in figure 8 were introduced into macrostructure, and the free deformation of a macrostructure was analyzed under a uniform electric field along macrostructural $x_{3}$ axis. Then material behaviours of the SEM EBSD-measured realistic microstructure were evaluated in response to the macroscopic external load.

The difference between single crystal and polycrystal is with and without the interference of material behaviours among neighbouring grains. Namely, grains show the various deformations according to their orientations and they have a large influence on others in polycrystalline microstructure. The interference of mechanical deformation under electrical loads appears obviously in piezoelectric energy. Therefore, we picked out the almost [001]-orientating grains from many grains in the microstructure as an example of computations, and investigated their piezoelectric energy. To be more precise, firstly we calculated the angle between crystallographic $c$ axis, which is the direction of spontaneous polarization in case of perovskite tetragonal structure, and microstructural $y_{3}$ axis for the microstructural finite element model. The elements whose angle between both axes is less than 5 degree were picked out as almost [001]-orienting grains and their piezoelectric energy was calculated from mechanical and electrical states cased by macrostructural external load. Figure 10 shows the relation between the piezoelectric energy and the average of orientation gap, which was calculated from neighbouring eight elements, in the realistic microstructure.

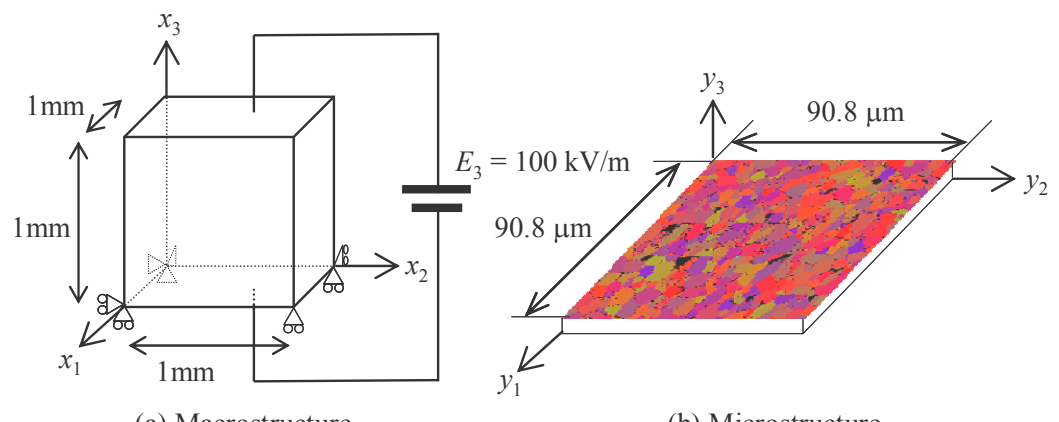

(a) Macrostructure

(b) Microstructure

Figure 9: The macrostructure, its boundary conditions and the microstructure used for the estimation of microscopic localized behaviours.

Additionally, the piezoelectric energy is compared with two specified states of [001]-orienting single crystal. One is a free deformation state and the other is a perfect-constraint state omnidirectionally under the same electric field with the 


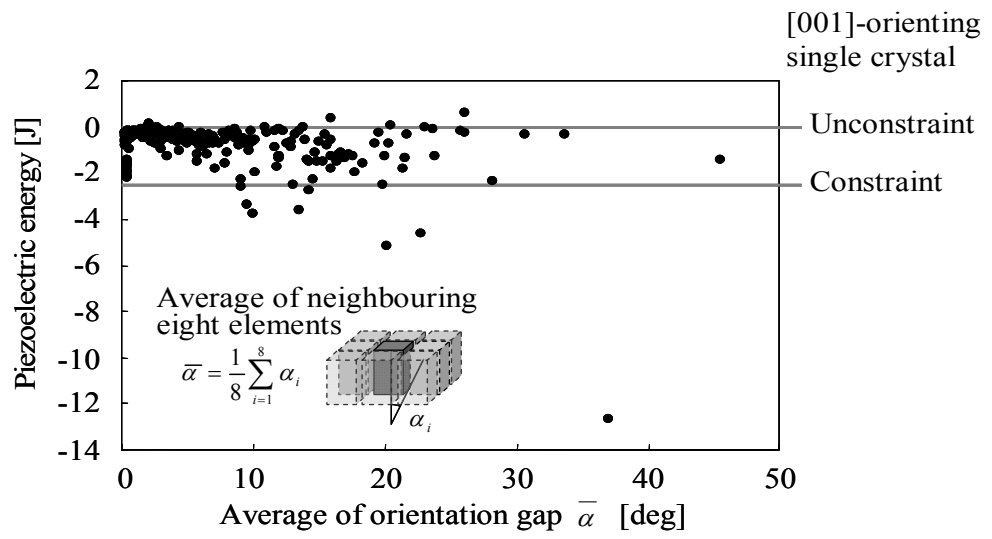

Figure 10: The relation between the piezoelectric energy and the average of orientation gap in the realistic microstructure.

polycrystal. This figure indicates that the piezoelectric energy of some grains reaches beyond one of perfect-constraint single crystal when the average of orientation gap is over 10 degree. However, there is no strong correlation between low piezoelectric energy and the orientation gap.

\section{Conclusions}

The realistic crystal morphology of a $\mathrm{BaTiO}_{3}$ polycrystalline piezoelectric material was obtained by SEM •EBSD measurement utilizing an amorphous osmium coating for the prevention of electrification. Then we employed a multiscale finite element simulation and investigated the effect of realistic crystal morphology on material properties and behaviours. As a computational result, the macroscopic homogenized material properties correspond approximately to experimental values and they satisfy the transverse isotropy. In a realistic polycrystal under electric field, there are some grains whose mechanical deformation is interfered more strongly by neighbouring grains compared with an omnidirectional-constraint state.

\section{Acknowledgements}

One of the authors (Y. Uetsuji) was financially supported by a Grant-in-Aid for Young Scientists (B) (No. 22760087) from the Ministry of Education, Culture, Sports, Science and Technology of Japan.

\section{References}

[1] Venables, J. \& Harland, C., Electron back scattering patterns - A new technique for obtaining crystallographic information in the scanning electron microscope. Philosophical Magazine, 27, pp.1193-1200, 1973. 
[2] Dingley, D. \& Randel, V., Microstructure determination by electron backscatter diffraction. Journal of Material Science, 27, pp.4545-4566, 1992.

[3] Wu, X., Pan, X. \& Stubbins, J.F., Analysis of notch strengthening of 316L stainless steel with and without irradiation-induced hardening using EBSD and FEM. Journal of Nuclear Materials, 361, pp.228-238, 2007.

[4] Calcagnotto, M., Ponge, D., Demir, E. \& Raabe, D., Orientation gradients and geometrically necessary dislocations in ultrafine grained dual-phase steels studied by 2D and 3D EBSD. Materials Science and Engineering: A, 527, pp.2738-2746, 2010.

[5] Yasutomi, Y. \& Takigawa, Y., Evaluation of crystallographic orientation analyses in ceramics by electron back scattering patterns (EBSP) method. Bulletin of the Ceramic Society of Japan, 37, pp.84-86, 2002.

[6] Yang, L.C., Dumler, I. \& Wayman, C.M., Studies of herringbone domain structures in lead titanate by electron back-scattering patterns. Materials Chemistry and Physics, 36, pp.282-288, 1994.

[7] Koblischka-Veneva, A. \& Mücklich, F., Orientation imaging microscopy applied to $\mathrm{BaTiO}_{3}$ ceramics. Crystal Engineering, 5, pp.235-242, 2002.

[8] Tai, C.W., Baba-kishi, K.Z. \& Wong, K.H., Microtexture characterization of PZT ceramics and thin films by electron microscopy. Micron, 33, pp.581-586, 2002.

[9] Gudes, J.M. \& Kikuchi, N., Preprocessing and postprocessing for materials based on the homogenization method with adaptive finite element methods. Computer Methods in Applied Mechanics and Engineering, 83, pp.143-198, 1990.

[10] Uetsuji, Y., Nakamura, Y., Ueda, S. \& Nakamachi, E., Numerical investigation on ferroelectric properties of piezoelectric materials. Modelling and Simulation in Materials Science and Engineering, 12, pp.S303-S317, 2004.

[11] Kuramae, H., Nishioka, H., Uetsuji, Y. \& Nakamachi, E., Development and performance evaluation of parallel iterative method. Transactions of the Japan Society for Computational Engineering and Science, Paper No.20070033, 2007.

[12] Uetsuji, Y., Yoshida, T., Yamakawa, T., Tsuchiya, K., Ueda, S. \& Nakamachi, E., Evaluation of ferroelectric properties of piezoelectric ceramics based on crystallographic homogenization method and crystal orientation analysis by SEM - EBSD technique. JSME International Journal Series A, 49, pp.209-215, 2006.

[13] Uetsuji, Y., Satou, Y., Nagakura, H., Nishioka, H., Kukamae, H. \& Tsuchiya, K., Crystal morphology analysis of piezoelectric ceramics using electron backscatter diffraction method and its application to multiscale finite element analysis. Journal of Computational Science and Technology, 2, pp.568-577, 2008.

[14] Jaffe, B., Piezoelectric ceramics, Academic Press: London and New York, p.74, 1971 . 